\title{
How does a simple enquiry compare to a detailed family history questionnaire to identify coronary heart disease or diabetic familial risk?
}

\author{
Miranda Wijdenes-Pijl, MSc ${ }^{1}$, Lidewij Henneman, PhD ${ }^{1,2}$, Laura Cross-Bardell, BA ${ }^{3}$, \\ Danielle R. M. Timmermans, $P h D^{l}$, and Nadeem Qureshi, MBBS, DM ${ }^{3}$
}

\begin{abstract}
Purpose: To examine whether a simple enquiry can provide similar family history information compared with a detailed questionnaire for coronary heart disease or diabetes. Methods: Data from two randomized controlled trials were extracted that assess the clinical value of using family history information for either coronary heart disease (ISRCTNI17943542) or diabetes risk assessment (NTR1938) in a community-based population. Outcome measures were percentage agreement, sensitivity, and specificity of self-reported family history for coronary heart disease and diabetes by means of a simple enquiry, when compared with a detailed questionnaire. Results: Agreement between both family history tools was $76.8 \%$ for first-degree relatives with coronary heart disease, and $89.2 \%$ and $87.6 \%$ for first- and seconddegree relatives with diabetes, respectively. The sensitivity was $44.2 \%$ for first-degree relatives with coronary heart disease, $81.9 \%$ for firstdegree relatives with diabetes, and $35.4 \%$ for second-degree relatives with diabetes. Specificity was $89.3 \%, 97.0 \%$, and $94.5 \%$, respectively. Conclusion: Compared with a detailed questionnaire, the simple enquiry correctly identified the majority of individuals classified as having no significant family history but missed a significant proportion of individuals with positive family history. Incorrect classification of family history, in particular the high false-negative rate, has implications on the utility of a simple enquiry in identifying familial risk in clinical practice. Genet Med 2011:13(5):443-446.
\end{abstract}

Key Words: family history, risk assessment, coronary heart disease, diabetes mellitus, sensitivity

A positive family history $(\mathrm{FH})$ is an independent risk factor for many common chronic diseases, such as cancer, cardiovascular diseases, and diabetes. ${ }^{1} \mathrm{FH}$ reflects shared genetic, behavioral, and environmental risk factors. FH is seen as a useful tool to select high-risk groups and may be used as a tool to raise awareness and target disease prevention in public health and primary care. ${ }^{2,3}$ Although reporting of the disease $\mathrm{FH}$ can be inaccurate, many people have a $\mathrm{FH}$ of common diseases. ${ }^{4} \mathrm{FH}$ assessments are part of the national professional guidelines for many common diseases, ${ }^{5}$ such as coronary heart disease (CHD)

From the Departments of ${ }^{1}$ Public and Occupational Health and ${ }^{2}$ Clinical Genetics, EMGO Institute for Health and Care Research, VU University Medical Center, Amsterdam, The Netherlands; and ${ }^{3}$ Division of Primary Care, University of Nottingham, Nottingham, United Kingdom.

Miranda Wijdenes-Pijl, MSc, Department of Public and Occupational Health, EMGO Institute for Health and Care Research, VU University Medical Center, PO Box 7057, Amsterdam 1007 MB, The Netherlands. E-mail: m.pijl@vumc.nl.

Disclosure: The authors declare no conflict of interest.

Submitted for publication September 2, 2010.

Accepted for publication November 22, 2010.

Published online ahead of print February 1, 2011.

DOI: $10.1097 /$ GIM.0b013e3182081fce and diabetes. However, FH is not systematically integrated into risk assessment of clinicians for these diseases in Europe or the United States, ${ }^{6,7}$ and there is, as yet, no standard method for taking $\mathrm{FH}$.

Comprehensive FH assessment can be achieved, e.g., by drawing a pedigree or family tree or by presenting questions in a tabular form. ${ }^{4}$ A detailed $\mathrm{FH}$ assessment is, however, time consuming, and nonspecialist clinicians, such as primary care practitioners, often have little time per patient. Familial risks, therefore, often go unrecognized, and avoidable risk is recognized too late. ${ }^{8,9}$ An alternative approach would be to minimize the detailed assessment to a simple enquiry as first screen for high risk without compromising the sensitivity of the familial risk assessment. The question is, however, whether a simple enquiry will have similar performance to a detailed FH assessment to identify significant FH of CHD and diabetes.

There is a great variation in methods used to collect $\mathrm{FH}$ across common diseases, ${ }^{1}$ although little is known about how the accuracy of FH is affected by the method of collection, e.g., paper based, web based, or in person and by simple enquiry or detailed questionnaire. ${ }^{10}$ Measures that have been used to evaluate adequacy of FH assessments include the percentage agreement, sensitivity, and specificity. ${ }^{4}$ Earlier studies indicate that there was reasonable agreement between self-completed $\mathrm{FH}$ questionnaires (FHQs) to identify familial cancer risk and measures of optimal approach (e.g., a detailed FH by a trained specialist) or criterion standard (e.g., FH recorded in relatives' charts) ${ }^{4}$ Currently, there are no studies comparing simple enquiry to a detailed assessment for common diseases, such as CHD or diabetes. The aim of this study is, therefore, to compare a simple enquiry of FH with a detailed assessment, which is considered to be the "optimal" or "best pragmatic" approach, rather than a criterion standard.

\section{MATERIALS AND METHODS}

In this study, assessing CHD or diabetic FH by means of a simple enquiry and a detailed questionnaire were compared. For this comparison, we conducted subanalyses within two cohorts derived from randomized trials that were conducted in two European countries with comparable health care systems. The first trial, the ADDFAM study, was conducted in the United Kingdom and assessed CHD FH. The second trial, the PreDiCT study, was conducted in the Netherlands and assessed diabetes FH. Both studies were approved by the Institutional Medical Research Ethics Committees, and informed consent of all participants was obtained.

\section{Study design \\ Coronary heart disease}

Subjects were participants of the ADDFAM study (ISRCTNI17943542) that assessed the clinical value of incorporating systematic FH information into CHD risk assessment 
in primary care. ${ }^{11}$ Patients between 30 and 65 years of age, who were offered cardiovascular disease risk assessment and referred for a cholesterol test as part of their normal care, either at their doctor's initiative or at their own request, were invited to participate. In line with the Joint British Societies guidelines, ${ }^{5} \mathrm{a}$ positive $\mathrm{FH}$ was defined as having at least one first-degree relative (mother, father, and sibling) with a CHD and an early age of onset (male $<55$ years; female $<65$ years). The FHQs that were used in this trial were as follows:

1. Simple enquiry: As part of the study questionnaire, the CHD FH was assessed by a simple enquiry: "Known family history of heart disease. Please tick box ONLY if you know the following is true. Either my father, or one or more of my brothers, has had heart disease before the age of 55 years/Either my mother, or one or more of my sisters, has had heart disease before the age of 65 years."

2. Detailed assessment: Detailed CHD FH information was collected by a CHD focused validated FHQ, an instrument that was shown to be effective to identify those with a FH of premature CHD and had acceptable face validity. ${ }^{12,13}$ Participants completed the FHQ before a risk assessment consultation with a primary care provider. The FHQ collects relevant medical history, medical information about close relatives (mother, father, siblings, children, and grandparents), information on more distant relatives, and information on age of onset of the diseases in a tabulated form. The FHQ enquired about heart disease, i.e., angina, heart attack, myocardial infarction, heart failure, blocked artery, and coronary artery disease.

The detailed FH assessment was assessed 6 months before the simple enquiry.

\section{Diabetes}

Subjects for the diabetic FH were participants of the PreDiCT study (NTR1938) that assessed the impact of diabetic familial risk information on self-reported risk-reducing behavior, by using a tailored web-based tool. Participants included were healthy people from the general population aged 35-65 years with an elevated risk for diabetes because of a body mass index $\geq 25 \mathrm{~kg} / \mathrm{m}^{2}$, recruited among an online panel maintained by an independent research agency. In line with a validated risk assessment tool for diabetes, the Diabetes Risk Test, ${ }^{14}$ a positive $\mathrm{FH}$ was defined as having at least one first-degree relative (mother, father, sibling, and children) with diabetes. As having a diabetic FH was an inclusion criterion in this trial, there was an oversampling of people with a FH. There were two ways of assessing diabetic $\mathrm{FH}$ in this trial:

1. Simple enquiry: As part of an online version of the Diabetes Risk Test, ${ }^{14}$ diabetic FH was assessed by a single item: "Does diabetes occur within your family? 1) no; 2) yes, with my grandfather, grandmother, uncle, aunt, nephew, niece; 3) yes, with my father, mother, brother, sister, or child."

2. Detailed assessment: Detailed FH was collected by means of a web-based systematic assessment. First, participants had to indicate the number of children and siblings, and the number of both paternal or maternal aunts and uncles. Subsequently, they could indicate for each first-degree relative and second-degree relative and whether these relatives had been diagnosed with diabetes or whether they did not know this.
The detailed FH assessment was assessed 3 months after the simple enquiry.

\section{Analyses}

The first metric assessed was the percentage agreement between both methods of FH collection, defined as the proportion of individuals identified by both approaches with a positive $\mathrm{FH}$ and with no significant FH among all participants, presented as a percentage. The discriminatory accuracy of the simple enquiry compared with the detailed assessment was assessed using metrics of sensitivity and specificity. Sensitivity was defined as the percentage of individuals who reported a positive FH based on the simple enquiry among all those who had a positive $\mathrm{FH}$ based on the detailed assessment and similarly for negative $\mathrm{FH}$ reports to calculate specificity. The complimentary metrics of false-negative rate and false-positive rate were also presented. The false-negative rate is 1-sensitivity and represents those with a positive FH on detailed assessment that were missed by simple enquiry, whereas the false-positive rate is 1-specificity and indicates those classified as having a positive $\mathrm{FH}$ on simple enquiry but no significant FH on detailed assessment.

\section{RESULTS}

\section{Characteristics of the study population}

Table 1 lists the characteristics of participants who completed the first FH assessment (this is the detailed assessment for the subgroup of people participating in the ADDFAM trial and the simple enquiry in the PreDiCT trial) and the characteristics of the respondents on the second FH assessment. Nonrespondents to the follow-up simple enquiry in the ADDFAM trial were significantly younger $(P<0.001)$ and more likely to be of non-white origin $(P<0.05)$. No further differences between respondents and nonrespondents on the second assessment were found in both trials.

\section{Comparison of simple enquiry with detailed assessment}

Table 2 presents the percentage agreement of FH obtained from a simple enquiry compared with a detailed assessment for CHD and diabetes. For first-degree relatives with $\mathrm{CHD}$, the agreement was 76.8\% (238/310). Agreement was 89.2\% (497/ $557)$ and $87.6 \%(488 / 557)$ for first- and second-degree relatives with diabetes, respectively. Sensitivity was $44.2 \%(38 / 86)$ for first-degree relatives with CHD, 81.9\% (236/288) for firstdegree relatives with diabetes, and 35.4\% (23/65) for seconddegree relatives with diabetes. False-negative rates on the single enquiry were $55.8 \%, 18.1 \%$, and $64.6 \%$, respectively. Specificity was $89.3 \%(200 / 224)$ for first-degree relatives with CHD, $97.0 \%(261 / 269)$ for first-degree relatives with diabetes, and 94.5\% (465/492) for second-degree relatives with diabetes. False-positive rates on the single enquiry were $10.7 \%, 3.0 \%$, and $5.5 \%$, respectively.

\section{DISCUSSION}

There was more than $75 \%$ agreement between the simple enquiry and a detailed questionnaire for assessing a $\mathrm{FH}$ of $\mathrm{CHD}$ or diabetes; however, the simple enquiry can give high falsenegative rates. In comparison with the detailed assessment, the simple enquiry correctly identified a greater proportion of individuals with no significant $\mathrm{FH}$ than individuals who had a positive $\mathrm{FH}$, i.e., higher specificity than sensitivity. When a simple FH enquiry is used as an initial screening tool, sensitivity 
Table 1. Characteristics of respondents who completed the first and second $\mathrm{FH}$ assessment

\begin{tabular}{|c|c|c|c|c|}
\hline & \multicolumn{2}{|c|}{ ADDFAM } & \multicolumn{2}{|c|}{ PreDiCT } \\
\hline & $\begin{array}{l}\text { Respondents completing } \\
\text { detailed assessment }{ }^{a} \text { : } \\
\text { baseline }(n=365)\end{array}$ & $\begin{array}{l}\text { Respondents completing } \\
\text { simple enquiry: 6-mo } \\
\text { follow-up }(n=310)^{b}\end{array}$ & $\begin{array}{l}\text { Respondents completing } \\
\text { simple enquiry }{ }^{a} \text { : } \\
\text { baseline }(n=584)\end{array}$ & $\begin{array}{l}\text { Respondents completing } \\
\text { detailed assessment: } \\
\text { 3-mo follow-up } \\
\quad(n=557)^{b}\end{array}$ \\
\hline Gender, $\%$ female & 52.3 & 54.5 & 48.5 & 49.4 \\
\hline Age $(\mathrm{yr})$, mean $\pm \mathrm{SD}$ & $50.3 \pm 9.4$ & $51.4 \pm 9.1$ & $53.4 \pm 5.6$ & $53.3 \pm 5.6$ \\
\hline Ethnicity, \% white ${ }^{c}$ & 94.0 & 95.2 & 97.4 & 97.3 \\
\hline Education, $\%$ higher education ${ }^{d}$ & 61.9 & 63.9 & 23.1 & 23.2 \\
\hline \multicolumn{5}{|c|}{$\begin{array}{l}{ }^{a} \text { For ADDFAM and PreDiCT trial, the response rate for the first assessment was } 98 \% \text { and } 90 \% \text {, respectively. } \\
\text { b } \\
\text { CFor ADDFAM and PreDiCT trial, the response rate for the second assessment was } 85 \% \text { and } 95 \% \text {, respectively. } \\
\text { cParticipants of the ADDFAM study were asked how they would describe their ethnic group, and participants of the PreDiCT study were asked to indicate their country } \\
\text { of birth. }\end{array}$} \\
\hline
\end{tabular}

Table 2. Percentage agreement, sensitivity, and specificity (with $95 \%$ confidence interval) of a simple enquiry of CHD or diabetes compared with a detailed assessment

\begin{tabular}{lccc}
\hline & $\begin{array}{c}\text { Agreement } \\
(95 \% \mathrm{CI})\end{array}$ & $\begin{array}{c}\text { Sensitivity } \\
(95 \% \mathrm{CI})\end{array}$ & $\begin{array}{c}\text { Specificity } \\
(95 \% \mathrm{CI})\end{array}$ \\
\hline Assessment of & & & \\
CHD first degree & $76.8(72.1-81.5)$ & $44.2(34.2-54.7)$ & $89.3(84.6-92.7)$ \\
Diabetes first degree & $89.2(86.6-91.8)$ & $81.9(77.1-86.0)$ & $97.0(94.2-98.5)$ \\
Diabetes second degree & $87.6(84.9-90.3)$ & $35.4(24.9-47.5)$ & $94.5(92.1-96.2)$ \\
\hline CI, confidence interval; CHD, coronary heart disease. & & & \\
\hline
\end{tabular}

is more important than specificity, as we do not want to miss individuals who have a positive FH (i.e., achieve low falsenegative rate). ${ }^{1}$ In a nonspecialist setting, it may be more acceptable for the simple enquiry to incorrectly identify some individuals with positive FHs. These cases will be excluded on a more detailed FH assessment, but the false negatives will be lost to follow-up. Taking this into account, the simple enquiry underperformed for its expected role.

Comparing the different conditions, the discriminatory accuracy for first-degree relatives was better for diabetes than for CHD simple enquiry. The ADDFAM and PreDiCT studies were not designed to assess the accuracy of FH collection against a criterion standard (e.g., recall by relatives, relatives' medical records, and death certificates) but designed to compare two approaches to assess FH. However, benchmarking the sensitivity against studies designed to assess criterion standard provides an indirect comparison. The Framingham Offspring Study, for example, shows higher sensitivity for paternal history reports of a heart attack below the age of 55 years $(74 \%)$ but lower sensitivity for diabetes $(56 \%),{ }^{15}$ compared with this study. Similarly, the NHLBI Family Heart Study, where age of onset was not identified, shows much greater sensitivity for CHD ( $85 \%$ ) but similar levels for diabetes $(87 \%) .{ }^{16}$ The findings for CHD in this study might be explained by the number of possible diagnoses that individuals have to consider when recalling relatives' heart disease diagnosis, compared with only one nonspecific disease diagnosis for diabetes. Also, recall error of the age of onset may complicate the accuracy of CHD FH reports. ${ }^{17}$ Adding more details to a single question might make it harder for respondents to understand. ${ }^{18}$ A possible solution is thus to break the single-question enquiry into a series of simple questions.

Some methodological drawbacks of this study need to be addressed. Although both samples used a community-based population, recruitment and assessment were different. The ADDFAM trial was performed in a primary care setting using paper-based questionnaires, whereas the PreDiCT trial was done in a public health setting using web-based questionnaires. Moreover, both trials had a different temporal sequence, where ADDFAM started with the detailed assessment and the PreDiCT trial first assessed the simple enquiry. There may have been better recall in the second assessment, and CHD simple enquiry would have performed better; however, the long time period between assessments may have limited this effect. The differences, however, will not obscure the conclusions, as the aim of this article is not to make a comparison between the CHD and diabetes samples but to examine whether a simple enquiry can provide similar FH information compared with a detailed questionnaire. Previous studies indicate that better educated individuals are more likely to complete FH collection. ${ }^{1}$ This selection bias was also noted in the ADDFAM trial but not in the PreDiCT trial.

Accurate FH reporting is essential if $\mathrm{FH}$ is used as a tool to target disease prevention in public health and primary care. ${ }^{2,3}$ Type of disease, the degree of family members, and the phrasing and details of the question could be important factors for an accurate FH assessment. The implication of the high rate of false-negative FH reports when based on a single enquiry is that a significant proportion of people at familial risk for CHD or diabetes will be missed. When assessing FH, either as part of a multifactorial risk assessment or to identify the single risk 
factor, the discriminatory accuracy of the simple enquiry needs to be taken into account when interpreting the findings.

\section{ACKNOWLEDGMENTS}

This study was performed as part of the Centre for Society and Genomics program that is funded by the Netherlands Genomics Initiative (PreDiCT) and the Department of Health Genetic Health Service Research program, United Kingdom (ADDFAM). This collaboration was supported by the EMGO Institute for Health and Care Research Travel Grant. The authors appreciate the constructive advice provided by Caroline Terwee, Department of Epidemiology and Biostatistics, VU University Medical Center, Amsterdam.

\section{REFERENCES}

1. Qureshi N, Wilson B, Santaguida P, et al. Family history and improving health. Evid Rep Technol Assess (Full Rep) 2009;186:1-135

2. Claassen L, Henneman L, Janssens AC, et al. Using family history information to promote healthy lifestyles and prevent diseases; a discussion of the evidence. BMC Public Health 2010;10:248

3. Yoon PW, Scheuner MT, Khoury MJ. Research priorities for evaluating family history in the prevention of common chronic diseases. Am J Prev Med 2003;24:128-135.

4. Qureshi N, Carroll JC, Wilson B, et al. The current state of cancer family history collection tools in primary care: a systematic review. Genet Med 2009; 11:495-506.

5. Wood D, Wray R, Poulter N, et al. JBS 2: Joint British Societies' guidelines on prevention of cardiovascular disease in clinical practice. Heart 2005;91: $1-52$.

6. Sheridan S, Pignone M, Mulrow C. Framingham-based tools to calculate the global risk of coronary heart disease: a systematic review of tools for clinicians. J Gen Intern Med 2003;18:1039-1052.
7. Reid GT, Walter FM, Brisbane JM, et al. Family history questionnaires designed for clinical use: a systematic review. Public Health Genomics 2009; $12: 73-83$.

8. Rich EC, Burke W, Heaton CJ, et al. Reconsidering the family history in primary care. J Gen Intern Med 2004;19:273-280.

9. Suther S, Goodson P. Barriers to the provision of genetic services by primary care physicians: a systematic review of the literature. Genet Med 2003;5: $70-76$.

10. Berg AO, Baird MA, Botkin JR, et al. National Institutes of Health Stateof-the-Science Conference Statement: family history and improving health. Ann Intern Med 2009;151:872-877.

11. Qureshi N, Armstrong S, Saukko P, et al. Realising the potential of the family history in risk assessment and primary prevention of coronary heart disease in primary care: ADDFAM study protocol. BMC Health Serv Res 2009;9:184.

12. Qureshi N, Bethea J, Modell B, et al. Collecting genetic information in primary care: evaluating a new family history tool. Fam Pract 2005;22:663669.

13. Qureshi N. Exploring the potential of the family history to identify genetic risk in primary care [dissertation]. Nottingham: Nottingham University, 2006.

14. Alssema M, Feskens EJ, Bakker SJ, et al. [Finnish questionnaire reasonably good predictor of the incidence of diabetes in The Netherlands]. Ned Tijdschr Geneeskd 2008;152:2418-2424.

15. Murabito JM, Nam BH, D'Agostino RB, et al. Accuracy of offspring reports of parental cardiovascular disease history: the Framingham Offspring Study. Ann Intern Med 2004;140:434-440.

16. Bensen JT, Liese AD, Rushing JT, et al. Accuracy of proband reported family history: the NHLBI Family Heart Study (FHS). Genet Epidemiol 1999; 17:141-150.

17. Toren K, Palmqvist M, Lowhagen O, et al. Self-reported asthma was biased in relation to disease severity while reported year of asthma onset was accurate. J Clin Epidemiol 2006;59:90-93.

18. Fowler FJ Jr. How unclear terms affect survey data. Public Opin $Q$ 1992; $56: 218-231$. 